\title{
QUELQUES SUITES EXACTES EN THÉORIE DES GENRES
}

\author{
par
}

\author{
Thong Nguyen Quang Do
}

\begin{abstract}
Résumé. - Nous donnons une démonstration cohomologique unifiée de diverses suites exactes et
\end{abstract} formules d'indice qui apparaissent dans la théorie classique des genres.

Abstract. - We give a unified cohomological proof of various exact sequences and index formulae which show up in classical genus theory.

\section{Table des matières}

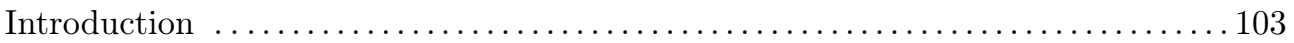

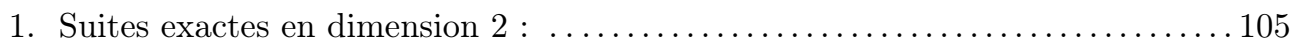

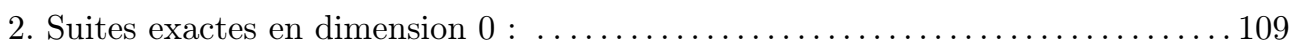

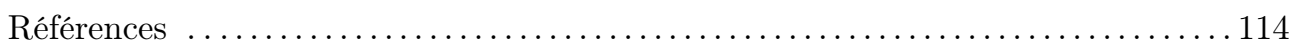

\section{Introduction}

Soit $F / k$ une extension galoisienne finie de corps de nombres, de groupe de Galois $G$. On adopte les notations usuelles :

$\mathcal{O}_{F}=$ l'anneau des entiers de $F, E_{F}=$ son groupe d'unités

$I_{F}=$ le groupe des idéaux, $P_{F}=F^{\times} / E_{F}$ le groupe des idéaux principaux, $C l_{F}=I_{F} / P_{F}=$ le groupe des classes d'idéaux de $F$

$J_{F}=$ le groupe des idèles, $C_{F}=J_{F} / F^{\times}$le groupe des classes d'idèles de $F$

$U_{F}=$ le groupe des idèles unités, i.e. des idèles de $F$ dont toutes les composantes sont des unités locales. Rappelons que par convention le groupe des unités en une place archimédienne est $\mathbb{R}^{\times}$ou $\mathbb{C}^{\times}$.

$P l_{F}=$ l'ensemble des places de $F$

$R(F / k)=$ l'ensemble des places de $k$ ramifiées dans $F$

Dans la suite on omettra $G$ dans l'écriture des groupes de $G$-cohomologie.

La théorie classique des genres étudie certains sous-modules remarquables de $C l_{F}$, les "genres" en question, qu'elle relie entre eux numériquement (par des formules d'indice) ou fonctoriellement (par des suites exactes). La multiplicité et la diversité des résultats à ce sujet (voir la bibliographie de [5], chapitre IV) pourraient donner une fausse impression de confusion et faire perdre de vue qu'en coulisses, les différentes approches reposent essentiellement sur le corps de classes. Mais il est vrai que le corps de classes possède plusieurs formulations - par les idéaux, par les idèles, par la cohomologie - la priorité accordée à l'une ou l'autre étant fonction de l'objectif à atteindre. Ainsi, dans [5], chap. IV, §4, l'approche par les rayons (au sens du corps de classes) donne une

Classification mathématique par sujets (2000). — 11R34,11R37. 
preuve synthétique des diverses "formules de genres", dans le cas le plus général possible d'une extension non forcément galoisienne, T-ramifiée et S-décomposée. Pour une extension galoisienne, l'approche cohomologique nous paraît naturellement la mieux adaptée à une démonstration unifiée de différentes "suites exactes de genres" telles que :

- la suite à huit termes de Dock Sang Rim (théorème principal de [10])

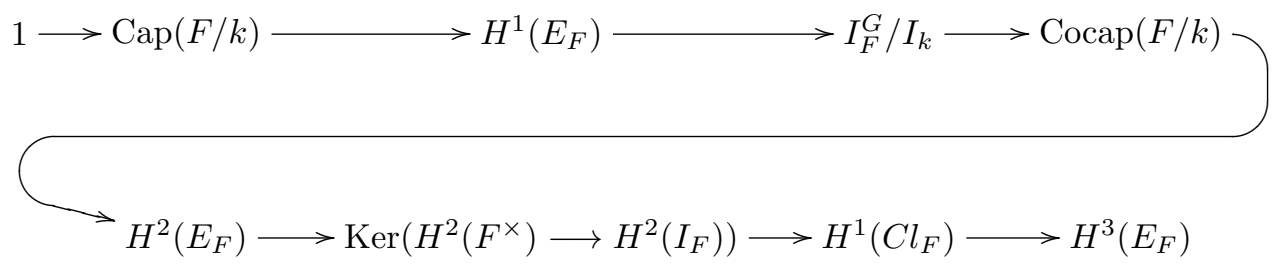

Ici $\operatorname{Cap}(F / k)$ et $\operatorname{Cocap}(F / k)$ désignent le noyau et le conoyau de l'homomorphisme d'extension $C l_{k} \longrightarrow C l_{F}^{G}$.

- la suite à sept termes d'Iwasawa ([6], propos. 1)

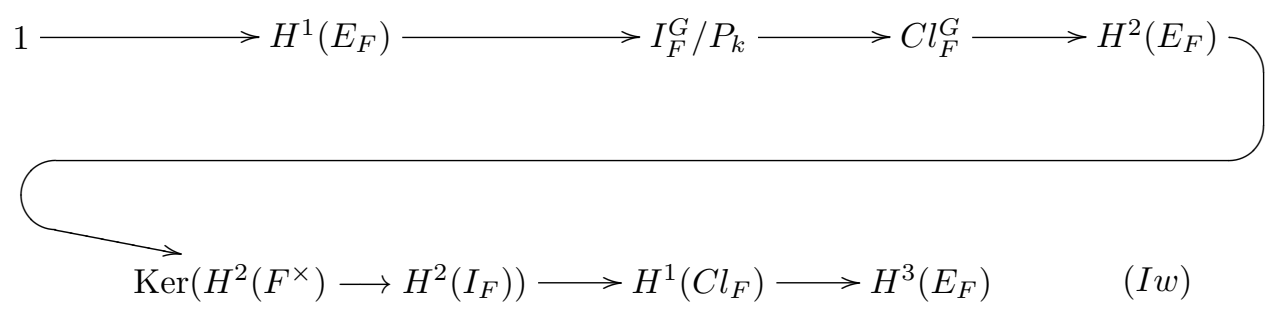

- la suite à trois termes de G. Gras ([5], IV, coroll. 4-4-2)

$$
1 \longrightarrow E_{k} / E_{k} \cap N J_{F} \longrightarrow \widehat{H}^{0}\left(U_{F}\right) \longrightarrow \operatorname{Gal}\left(F_{h}^{a b} / k_{h}\right) \longrightarrow 1
$$

Ici $F_{h}\left(\right.$ resp. $\left.k_{h}\right)$ désigne le corps de Hilbert de $F\left(\right.$ resp. $k$ ) et $F_{h}^{a b}$ la sous-extension abélienne maximale de $F_{h} / k$.

- la suite à sept termes récemment obtenue par Karoubi \& Lambre ([8])

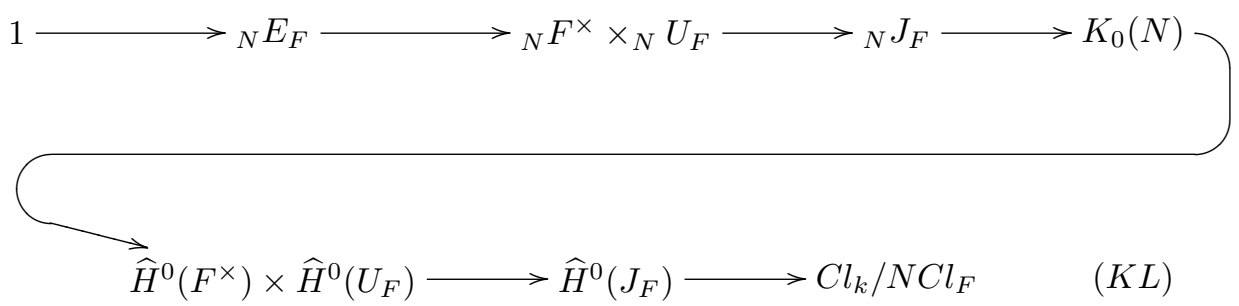

Ici $N$ désigne la norme de $F / k,{ }_{N}($.$) le noyau de cette norme et K_{0}(N)$ le groupe $K_{0}$ du foncteur "restriction des scalaires" de la catégorie $\operatorname{Proj}\left(\mathcal{O}_{F}\right)$ des $\mathcal{O}_{F}$-modules projectifs de type fini vers la catégorie correspondante $\operatorname{Proj}\left(\mathcal{O}_{k}\right)$.

Le présent exposé ne prétend à aucune originalité, si ce n'est son approche synthétique qui permet de retrouver et de relier entre elles toutes les suites exactes précitées, par la seule cohomologie d'un unique diagramme commutatif, par ailleurs classiquement connu, et qui résume les relations entre 
classes d'idèles et d'idéaux :

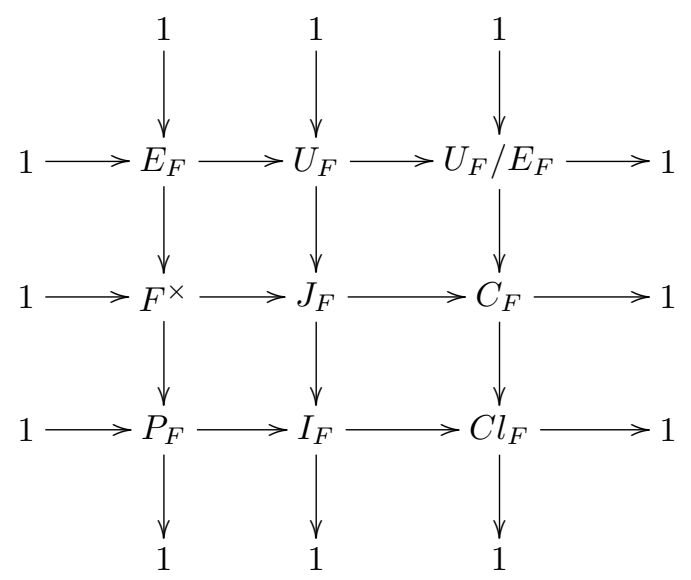

Remarque : Dans cet exposé, on s'est placé dans le cadre classique de la théorie des genres, mais on a reculé devant la tâche de l'élargir au cadre T-ramifié S-décomposé (comme c'est fait au chap. IV de [5], mais dans le langage des rayons). Comme les arguments employés ici reposent exclusivement sur le formalisme cohomologique, ils doivent pouvoir s'étendre tels quels. D'ailleurs [10] et [6] traitent du cas T-ramifié. Pour un premier pas vers la $T-S$ capitulation voir [9].

\section{Suites exactes en dimension 2 :}

Les deux suites exactes (DSR) et (Iw) citées dans l'introduction possèdent une partie commune à droite du terme $H^{2}\left(E_{F}\right)$ alors que les parties à gauche semblent a priori différentes. On va faire une étude séparée.

1.1. La partie droite de (DSR) et (Iw) :- Par cohomologie en dimensions 1, 2, 3, le diagramme (1) donne immédiatement un autre diagramme commutatif exact :

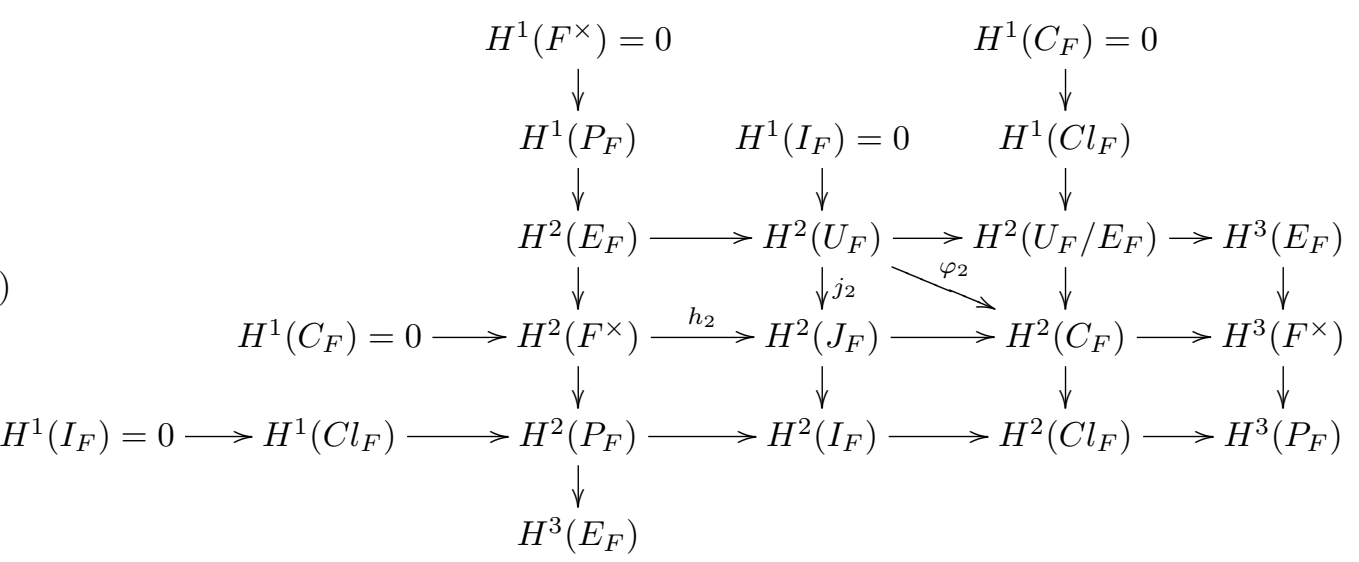

La nullité de $H^{1}\left(F^{\times}\right)$est le théorème 90 de Hilbert. Celle de $H^{1}\left(I_{F}\right)$ s'obtient par exemple en appliquant le lemme de Shapiro; on notera $j_{2}$ la flèche injective $H^{2}\left(U_{F}\right) \longrightarrow H^{2}\left(J_{F}\right)$. Enfin la nullité de $H^{1}\left(C_{F}\right)$ est un résultat de formation de classes; l'injectivité subséquente de la flèche $h_{2}: H^{2}\left(F^{\times}\right) \longrightarrow H^{2}\left(J_{F}\right)$ est parfois appelée le principe de Hasse cohomologique. Posons

$$
\begin{gathered}
V_{2}=j_{2}^{-1}\left(j_{2}\left(H^{2}\left(U_{F}\right)\right) \cap h_{2}\left(H^{2}\left(F^{\times}\right)\right)\right) \\
W_{2}=h_{2}^{-1}\left(j_{2}\left(V_{2}\right)\right)=\operatorname{Ker}\left(H^{2}\left(F^{\times}\right) \longrightarrow H^{2}\left(I_{F}\right)\right)
\end{gathered}
$$

En suivant le diagramme (2), on voit facilement que :

(i) dans la première colonne, la flèche $H^{2}\left(E_{F}\right) \longrightarrow H^{2}\left(F^{\times}\right)$tombe dans $W_{2}$

(ii) dans la première ligne, la flèche $H^{2}\left(E_{F}\right) \longrightarrow H^{2}\left(U_{F}\right)$ tombe dans $V_{2}$ 
(iii) dans le carré gauche supérieur, $h_{2}\left(W_{2}\right)=j_{2}\left(V_{2}\right)$. On pourrait noter ce module $H^{2}\left(F^{\times}\right) \cap$ $H^{2}\left(U_{F}\right)$ (comme dans [8], mais c'est un abus dangereux). Le résultat suivant complète la partie droite de $[\mathbf{1 0}]$ et $[\mathbf{6}]$ :

Théorème 1.1. - On a deux suites exactes isomorphes :

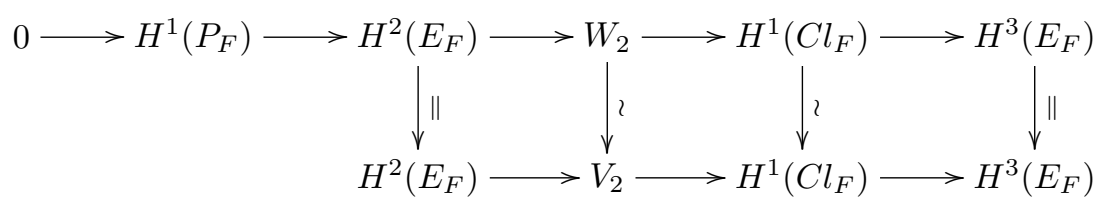

Preuve : la suite exacte supérieure est la partie droite commune à $(D S R)$ et $(I w)$. C'est en fait la première colonne de (2), en tenant compte de la remarque (i) ci-dessus. De même, la suite exacte inférieure est la première ligne de (2), en tenant compte de la remarque (ii). Il reste à construire les isomorphismes verticaux. Il s'agit en quelque sorte de "rabattre" l'une sur l'autre la première colonne et la première ligne du diagramme (2) :

- l'isomorphisme $W_{2} \stackrel{\sim}{\longrightarrow} V_{2}$ provient de la remarque (iii)

- la construction de l'isomorphisme $H^{1}\left(C l_{F}\right) \stackrel{\sim}{\longrightarrow} H^{1}\left(C l_{F}\right)$ va se faire de façon analogue. Pour distinguer entre les deux copies de $H^{1}\left(C l_{F}\right)$, revenons aux définitions :

$H^{1}\left(C l_{F}\right)=\operatorname{Ker}\left(H^{2}\left(P_{F}\right) \longrightarrow H^{2}\left(I_{F}\right)\right)$ dans la suite exacte supérieure et $H^{1}\left(C l_{F}\right)=\operatorname{Ker}\left(H^{2}\left(U_{F} / E_{F}\right) \longrightarrow H^{2}\left(C_{F}\right)\right)$ dans la suite inférieure. On va faire tous les calculs en passant par $J_{F} / E_{F}$. Examinons le premier noyau : l'homomorphisme $P_{F}=F^{\times} / E_{F} \longrightarrow I_{F}=$ $J_{F} / U_{F}$ est le composé de deux homomorphismes $F^{\times} / E_{F} \longrightarrow J_{F} / E_{F}$ et $J_{F} / E_{F} \longrightarrow J_{F} / U_{F}$. Or la suite exacte $1 \rightarrow F^{\times} / E_{F} \rightarrow J_{F} / E_{F} \rightarrow C_{F} \rightarrow 1$ donne par cohomologie une suite $H^{1}\left(C_{F}\right)=$ $0 \rightarrow H^{2}\left(P_{F}\right) \stackrel{h_{F}}{\rightarrow} H^{2}\left(J_{F} / E_{F}\right) \rightarrow H^{2}\left(C_{F}\right) \cdots$ De même, la suite exacte $1 \rightarrow U_{F} / E_{F} \rightarrow J_{F} / E_{F} \rightarrow$ $J_{F} / U_{F} \rightarrow 1$ donne par cohomologie $H^{1}\left(I_{F}\right)=0 \rightarrow H^{2}\left(U_{F} / E_{F}\right) \stackrel{j_{1}}{\rightarrow} H^{2}\left(J_{F} / E_{F}\right) \rightarrow H^{2}\left(I_{F}\right) \cdots$ Donc un élément $x$ de $H^{2}\left(P_{F}\right)$ est dans $\operatorname{Ker}\left(H^{2}\left(P_{F}\right) \rightarrow H^{2}\left(I_{F}\right)\right)$ si et seulement si $h_{1}(x)$ est dans l'image de $j_{1}$; autrement dit,

$$
\operatorname{Ker}\left(H^{2}\left(P_{F}\right) \rightarrow H^{2}\left(I_{F}\right)\right)=h_{1}^{-1}\left(h_{1}\left(H^{2}\left(P_{F}\right)\right) \cap j_{1}\left(H^{2}\left(U_{F} / E_{F}\right)\right)\right)
$$

Exactement de la même façon,

$$
\operatorname{Ker}\left(H^{2}\left(U_{F} / E_{F}\right) \rightarrow H^{2}\left(C_{F}\right)\right)=j_{1}^{-1}\left(h_{1}\left(H^{2}\left(P_{F}\right)\right) \cap j_{1}\left(H^{2}\left(U_{F} / E_{F}\right)\right)\right) .
$$

On en déduit évidemment un isomorphisme $H^{1}\left(C l_{F}\right) \stackrel{\sim}{\longrightarrow} H^{1}\left(C l_{F}\right)$ qui est compatible avec l'isomorphisme précédent $W_{2} \stackrel{\sim}{\longrightarrow} V_{2}$.

\section{Remarques :}

1) Les constructions précédentes cachent manifestement des calculs sur des produits fibrés. Voir le $\S 2.5$ ci-après.

2) Dans le cas particulier où $G$ est cyclique, on peut arrêter à droite les suites exactes du théorème 1.1 Voir le théorème 2.2 ci-après.

La définition de $V_{2}$ comme une intersection n'est pas forcément des plus commodes quand il s'agit de faire des calculs d'ordre ou d'indice. Heureusement le corps de classes permet de "linéariser" le problème. Fixons d'abord quelques notations locales :

Pour toute place $v$ de $k$, on choisit arbitrairement une place $w$ de $F$ au-dessus de $v$ et l'on note $G_{w}=\operatorname{Gal}\left(L_{w} / k_{v}\right)$ le groupe de décomposition correspondant. On omettra $G_{w}$ dans l'écriture des groupes de cohomologie. Soit $U_{w}$ le groupe des unités de $L_{w}$. Alors $H^{2}\left(U_{F}\right)=\bigoplus_{v \in P l_{k}} H^{2}\left(U_{w}\right)$, mais il est bien connu que $H^{2}\left(U_{w}\right)=0$ si la place $w$ est non ramifiée au-dessus de $v$, de sorte que $H^{2}\left(U_{F}\right)=\bigoplus_{v \in R} H^{2}\left(U_{w}\right)$, où $R=R(F / k)$ désigne l'ensemble des places (finies ou infinies) de $k$ qui se ramifient dans $F$. Alors : 
Proposition 1.2. - Dans le diagramme (2), notons $\varphi_{2}$ l'homomorphisme composé

$$
H^{2}\left(U_{F}\right) \longrightarrow H^{2}\left(J_{F}\right) \longrightarrow H^{2}\left(C_{F}\right) .
$$

Alors $V_{2}=\operatorname{Ker} \varphi_{2}, \operatorname{Im} \varphi_{2}$ est cyclique, et

$$
\left|V_{2}\right|=\frac{\prod_{v \in R} d_{v}}{\underset{v \in R}{\operatorname{ppcm}\left(d_{v}\right)},}
$$

ò̀ $d_{v}=\left|H^{2}\left(U_{w}\right)\right|$.

Preuve : En suivant le diagramme (2), on voit immédiatement que $V_{2}=\operatorname{Ker} \varphi_{2}$. Le corps de classes permet de "linéariser" $\varphi_{2}$. En effet,

$$
H^{2}\left(C_{F}\right) \cong \frac{1}{|G|} \mathbb{Z} / \mathbb{Z} \quad \text { et } \quad H^{2}\left(L_{w}^{\times}\right) \cong \frac{1}{\left|G_{w}\right|} \mathbb{Z} / \mathbb{Z}
$$

de manière canonique, et en faisant ces identifications, l'application naturelle

$$
H^{2}\left(J_{F}\right) \longrightarrow H^{2}\left(C_{F}\right)
$$

n'est autre que l'application "somme des composantes" (voir e.g. [2], chap.VII). Or la suite exacte de valuation $1 \rightarrow U_{w} \rightarrow L_{w}^{\times} \rightarrow \mathbb{Z} \rightarrow 0$ montre que $H^{2}\left(U_{w}\right) \hookrightarrow H^{2}\left(L_{w}^{\times}\right)$(c'est une preuve possible de l'injectivité de $H^{2}\left(U_{F}\right) \longrightarrow H^{2}\left(J_{F}\right)$ dans le diagramme $\left.(2)\right)$, de sorte que $\varphi_{2}$ s'identifie à l'application "somme" :

$$
\bigoplus_{v \in R} \frac{1}{d_{v}} \mathbb{Z} / \mathbb{Z} \longrightarrow \frac{1}{|G|} \mathbb{Z} / \mathbb{Z}
$$

Les assertions de la proposition 1.2 s'ensuivent immédiatement.

Remarque : En une place $v$ totalement ramifiée, $d_{v}$ est égal au degré local ([11] chap.12, $§ 1$, exercice 2).

1.2. Les parties gauches de $(D S R)$ et $(I w)$ :- Compléter vers la gauche les suites exactes du théo.1.1 revient à résoudre (par des suites exactes) le noyau $H^{1}\left(P_{F}\right)$. C'est fait dans [10] et [6] en manipulant élémentairement les définitions des idéaux principaux et des classes d'idéaux :

$$
1 \longrightarrow E_{F} \longrightarrow F^{\times} \longrightarrow P_{F} \longrightarrow 1
$$

et

$$
1 \longrightarrow P_{F} \longrightarrow I_{F} \longrightarrow C l_{F} \longrightarrow 1
$$

Les résolutions obtenues sont distinctes mais pas indépendantes. Plus précisément :

Proposition 1.3. - On a un diagramme commutatif aux lignes exactes :

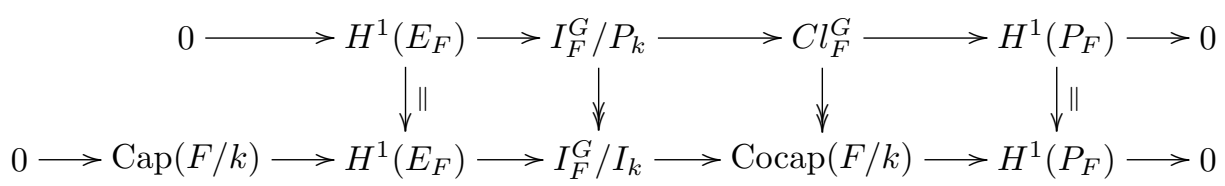

Preuve : La suite exacte (3) donne par cohomologie :

$$
1 \longrightarrow E_{F}^{G} \longrightarrow\left(F^{\times}\right)^{G} \longrightarrow P_{F}^{G} \longrightarrow H^{1}\left(E_{F}\right) \longrightarrow 0,
$$

d'où un isomorphisme $P_{F}^{G} / P_{k} \stackrel{\sim}{\longrightarrow} H^{1}\left(E_{F}\right)$. De son côté, la suite exacte (4) donne :

$$
1 \longrightarrow P_{F}^{G} \longrightarrow I_{F}^{G} \longrightarrow C l_{F}^{G} \longrightarrow H^{1}\left(P_{F}\right) \longrightarrow 0
$$

On peut transformer cette dernière suite de deux manières :

- soit l'écrire sous la forme :

$$
1 \longrightarrow P_{F}^{G} / P_{k} \longrightarrow I_{F}^{G} / P_{k} \longrightarrow C l_{F}^{G} \longrightarrow H^{1}\left(P_{F}\right) \longrightarrow 0
$$

pour obtenir la partie gauche de $(I w)$ 
- soit la mettre dans un diagramme commutatif :

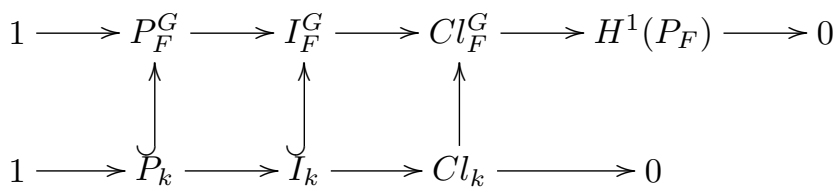

d'où, par le lemme du serpent, la partie gauche de $(D S R)$

Une application immédiate est la formule des classes ambiges de Chevalley (c'est pratiquement la même démonstration que l'originale) :

Corollaire 1.4. - Notons $\theta_{2}$ l'homomorphisme naturel $H^{2}\left(E_{F}\right) \longrightarrow H^{2}\left(U_{F}\right)$. Alors :

$$
\left|C l_{F}^{G}\right|=\frac{\left|C l_{k}\right|}{\left|\operatorname{Im} \theta_{2}\right|} \frac{\left|H^{2}\left(E_{F}\right)\right|}{\left|H^{1}\left(E_{F}\right)\right|} \prod_{v \in R^{0}} e_{v}
$$

Ici $R^{0}=R^{0}(F / k)$ désigne l'ensemble des places finies de $k$ ramifiées dans $F$, et $e_{v}$ est l'indice de ramification en $v$. Dans le cas particulier où $G$ est cyclique, cette formule devient :

$$
\left|C l_{F}^{G}\right|=\frac{\left|C l_{k}\right|}{\left(E_{k}: E_{k} \cap N J_{F}\right)} \frac{\prod_{v \in R^{0}} e_{v}}{[F: k]} 2^{a},
$$

où a est le nombre de places réelles de $k$ qui se complexifient dans $F$ et $N$ est la norme de $F$ à $k$.

Preuve : Notons que $\frac{\left|C l_{F}^{G}\right|}{\left|C l_{k}\right|}=\frac{|\operatorname{Cocap}(F / k)|}{|\operatorname{Cap}(F / k)|}$ et que $I_{F}^{G} / I_{k} \simeq \bigoplus_{v \in R^{0}} \mathbb{Z} / e_{v} \mathbb{Z}$. Il suffit alors de prendre le produit alterné des ordres dans la partie gauche de $(D S R)$ pour obtenir la première formule. Si $G$ est cyclique, le quotient de Herbrand $\left|\widehat{H}^{0}\left(E_{F}\right)\right| /\left|H^{1}\left(E_{F}\right)\right|$ est connu (voir e.g. [2], p.178) et $\theta_{2}$ s'identifie à l'application naturelle $\widehat{H}^{0}\left(E_{F}\right) \longrightarrow \widehat{H}^{0}\left(J_{F}\right)$. On en déduit aisément la seconde formule.

Remarque : Le théorème 1.1 donne a priori une troisième résolution de $H^{1}\left(P_{F}\right)$, obtenue en prolongeant à gauche la suite exacte inférieure :

$$
1 \rightarrow U_{k} / E_{k} \rightarrow\left(U_{F} / E_{F}\right)^{G} \rightarrow H^{1}\left(E_{F}\right) \rightarrow H^{1}\left(U_{F}\right) \rightarrow H^{1}\left(U_{F} / E_{F}\right) \rightarrow H^{1}\left(P_{F}\right) \rightarrow 0
$$

Mais en fait on n'obtient pas grand chose de nouveau par rapport à $(D S R)$. En effet, le début de l'avant-dernière colonne de (2) donne un diagramme commutatif aux lignes exactes :

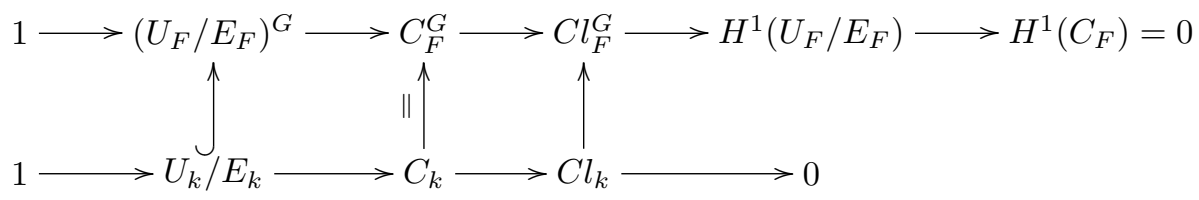

d'où, par le lemme du serpent : $\operatorname{Cap}(F / k) \simeq \operatorname{Coker}\left(U_{k} / E_{k} \longrightarrow\left(U_{F} / E_{F}\right)^{G}\right)$ et $\operatorname{Cocap}(F / k) \simeq$ $H^{1}\left(U_{F} / E_{F}\right)$. On en déduit aussi $H^{1}\left(U_{F}\right) \simeq I_{F}^{G} / I_{k}$, ce qui pouvait se voir directement par localisation. Pour obtenir des renseignements vraiment nouveaux, il va falloir remplacer la cohomologie ordinaire par celle de Tate. 


\section{Suites exactes en dimension 0 :}

Réécrivons d'abord l'analogue du diagramme (2) en dimensions -1, 0, 1 :

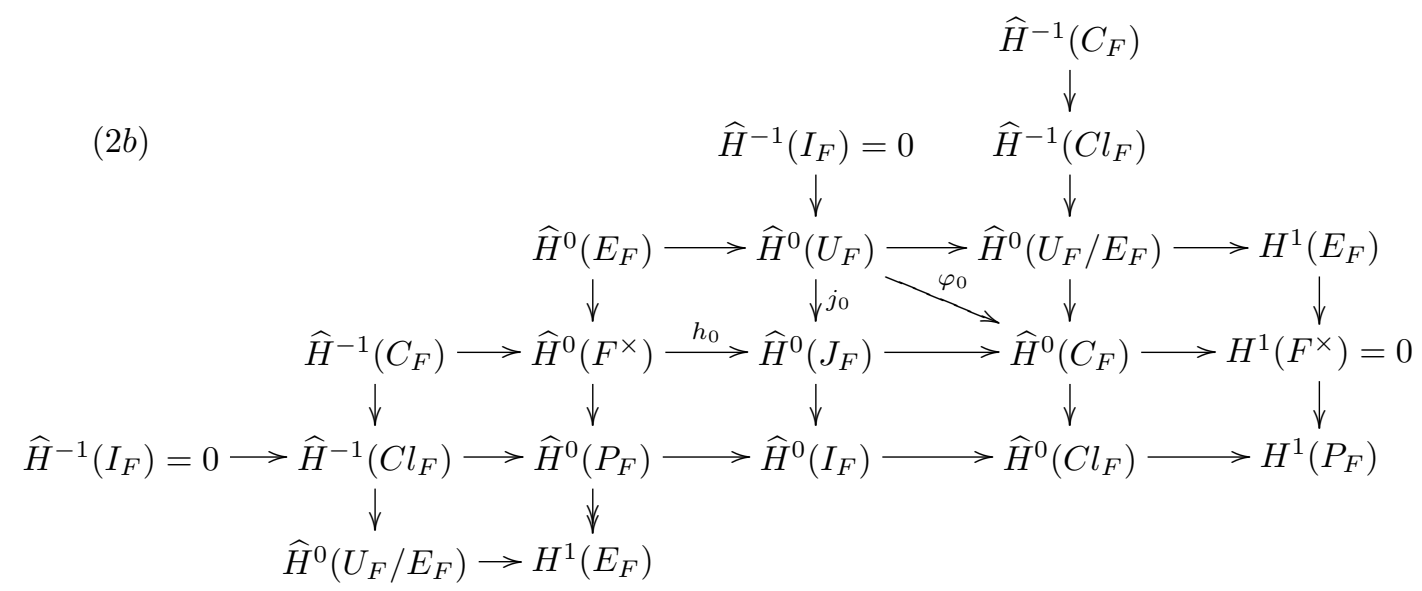

La nullité de $\widehat{H}^{-1}\left(I_{F}\right)$ se voit par exemple en appliquant le lemme de Shapiro. Un point-clé va consister à raccourcir la colonne de droite en faisant intervenir certains genres.

2.1. Divers genres :- Dans la suite, on notera $F_{h}$ le corps de Hilbert de $F$ et l'on identifiera, par le corps de classes, $C l_{F}$ à $H_{F}:=\operatorname{Gal}\left(F_{h} / F\right)$. D'une façon un peu vague, un genre est un sous$G$-module remarquable (du point de vue arithmétique) de $H_{F}$. Dans la littérature, on rencontre classiquement :

- le genre central $H_{c}=I_{G} H_{F}$, où $I_{G}$ désigne l'idéal d'augmentation de $G$. Le corps $F_{c}$ fixé par $H_{c}$ est la surextension centrale maximale de $F / k$ contenue dans $F_{h}$.

- les noyaux ${ }_{\nu} H_{F}$ et ${ }_{N} H_{F}$ de la norme algébrique $\nu=\sum_{\sigma \in G} \sigma$ et de la norme arithmétique $N: C l_{F} \longrightarrow C l_{k}$. Il est clair que $N$ induit un morphisme injectif ${ }_{\nu} C l_{F} /{ }_{N} C l_{F} \hookrightarrow \operatorname{Cap}(F / k)$.

- le genre principal galoisien $H_{f}$ ( $f$ pour Fröhlich), qui est le fixateur du "corps des genres" de Fröhlich $F_{f}$, défini comme étant la sous-extension maximale de $F_{h}$ qui s'obtient en composant $F$ avec une extension abélienne de $k$.

- le genre principal arithmétique $H_{g}$ ( $g$ pour Gauss), qui est la généralisation en termes de symboles normiques du genre originellement introduit par Gauss pour les corps quadratiques. Nous en rappelons brièvement la définition suivant [3]. Pour toute place $v$ de $k$, on a l'application de réciprocité $\chi_{v}: k^{\times} \longrightarrow \widehat{H}^{0}\left(F_{w}^{\times}\right) \simeq G_{w}^{a b}\left(=\right.$ l'abélianisé de $\left.G_{w}\right)$, et l'on pose $\chi(a)=\left(\chi_{v}(a)\right)_{v \in R}$. Soit $S=\chi\left(E_{k}\right)$. Toute classe $[\mathfrak{A}] \in_{N} C l_{F}$ est représentée par un idéal $\mathfrak{A}$ tel que $N(\mathfrak{A})$ est un idéal principal $(a) \in P_{k}$, et l'on vérifie que l'homomorphisme

$$
\begin{array}{r}
\psi: \quad{ }_{N} H_{F} \longrightarrow\left(\bigoplus_{v \in R} G_{w}^{a b}\right) / S \\
{[\mathfrak{A}] \longmapsto \chi(\mathfrak{A}) \bmod S}
\end{array}
$$

est bien défini. Alors $H_{g}:=\operatorname{Ker} \psi$. Le résultat principal de [3] est l'égalité des genres principaux : $H_{g}=H_{f}$. Sa preuve utilise les symboles normiques et la formulation du corps de classes en termes d'idéaux. On redonnera ci-dessous une démonstration cohomologique dans le style du §1. 
Du point de vue galoisien, les relations entre les divers genres sont résumées dans le diagramme d'extensions suivant :

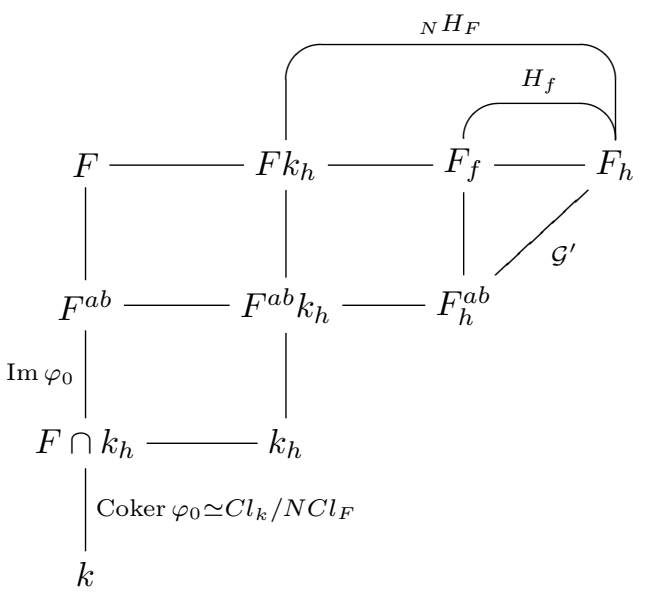

Quelques explications :

- si $\mathcal{G}=\operatorname{Gal}\left(F_{h} / k\right)$, le sous-groupe dérivé $\mathcal{G}^{\prime}$ fixe $F_{h}^{a b}$, la sous-extension abélienne maximale de $F_{h} / k$. Donc $F_{f}=F F_{h}^{a b}$ et $H_{f}=\mathcal{G}^{\prime} \cap H_{F}$.

- d'après les propriétés fonctorielles du symbole d'Artin par rapport à la norme, il est immédiat que $N C l_{F} \simeq \operatorname{Gal}\left(k_{h} / k_{h} \cap F\right)$, et donc ${ }_{N} C l_{F} \simeq \operatorname{Gal}\left(F_{h} / F k_{h}\right)$ et $C l_{k} / N C l_{F} \simeq \operatorname{Gal}\left(k_{h} \cap F / k\right)$.

- de façon analogue à $\varphi_{2}$ dans le $\S 1$, introduisons l'homomorphisme composé $\varphi_{0}: \widehat{H}^{0}\left(U_{F}\right) \longrightarrow$ $\widehat{H}^{0}\left(J_{F}\right) \longrightarrow \widehat{H}^{0}\left(C_{F}\right)$. Par le corps de classes local, $\widehat{H}^{0}\left(U_{F}\right) \simeq \bigoplus_{v \in R}\left(G_{w}^{a b}\right)^{i n}$, où $\left(G_{w}^{a b}\right)^{i n}$ est le sousgroupe d'inertie de $\left(G_{w}^{a b}\right)$; par le corps de classes global, $\widehat{H}^{0}\left(C_{F}\right) \simeq G^{a b}$. Modulo ces identifications, $\varphi_{0}\left(\left(\sigma_{w}\right)\right)=\prod_{w \in R} \sigma_{w}$, donc $\operatorname{Im} \varphi_{0}$ fixe la sous-extension non ramifiée maximale de $F^{a b}$, qui est $F \cap k_{h}$, et l'on a bien $\operatorname{Gal}\left(F \cap k_{h} / k\right) \simeq \operatorname{Coker} \varphi_{0}$.

2.2. Deux suites fondamentales :- Il est facile de voir que $H_{f} \supset H_{c}$, et $H_{f}=H_{c}$ si $G$ est cyclique. Dans le cas général, une interprétation cohomologique de $H_{f} / H_{c}$ a été donnée par Artin-Tate-Gold en utilisant la classe fondamentale de $H^{2}\left(C_{F}\right)$ et le théorème de Safarevic-Weil :

Lemme 2.1. - L'image de $\widehat{H}^{-1}\left(C_{F}\right) \longrightarrow \widehat{H}^{-1}\left(C l_{F}\right)$ est isomorphe à $H_{f} / H_{c}$, et donc le début de la colonne de droite du diagramme (2b) peut s'écrire:

$$
0 \longrightarrow{ }_{\nu} H_{F} / H_{f} \longrightarrow \widehat{H}^{0}\left(U_{F} / E_{F}\right) \longrightarrow \widehat{H}^{0}\left(C_{F}\right)
$$

Preuve : voir [1], chapitre XIII, théorème 3 et [4], proposition 7.

De manière analogue au $\S 1$, on peut poser :

$$
\begin{aligned}
& V_{0}=j_{0}^{-1}\left(j_{0}\left(\widehat{H}^{0}\left(U_{F}\right)\right) \cap h_{0}\left(\widehat{H}^{0}\left(F^{\times}\right)\right)\right)=\operatorname{Ker} \varphi_{0} \\
& W_{0}=h_{0}^{-1}\left(j_{0}\left(V_{0}\right)\right)=\operatorname{Ker}\left(\widehat{H}^{0}\left(F^{\times}\right) \longrightarrow \widehat{H}^{0}\left(I_{F}\right)\right)
\end{aligned}
$$

La seconde colonne du diagramme (2b) s'écrit alors :

$$
\cdots \longrightarrow \widehat{H}^{0}\left(E_{F}\right) \longrightarrow W_{0} \longrightarrow{ }_{\nu} H_{F} / H_{c} \longrightarrow H^{1}\left(E_{F}\right) \longrightarrow \cdots
$$

et la première ligne, en tenant compte du lemme 2.1 :

$$
\cdots \longrightarrow \widehat{H}^{0}\left(E_{F}\right) \longrightarrow V_{0} \longrightarrow{ }_{\nu} H_{F} / H_{f} \longrightarrow H^{1}\left(E_{F}\right) \longrightarrow \cdots
$$

Le résultat central de cette section est le 
Théorème 2.2. - On a un diagramme commutatif aux lignes exactes :

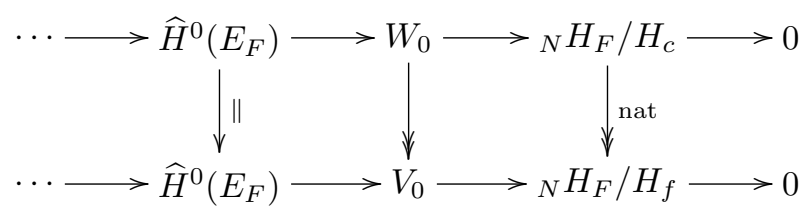

Preuve : Dans le diagramme (2b), le carré commutatif contenant les homomorphismes $h_{0}$ et $j_{0}$ donne immédiatement une flèche surjective $W_{0} \rightarrow V_{0}$. En remarquant que la première colonne à gauche coïncide avec l'avant-dernière colonne à droite, on en déduit facilement un diagramme commutatif :

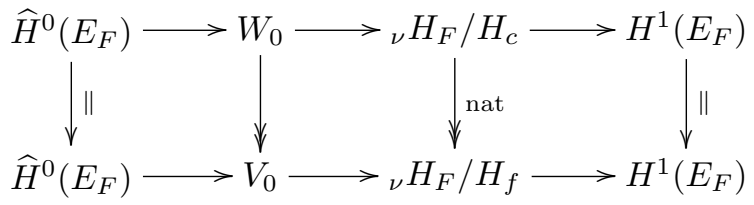

Supposons momentanément que nous ayons démontré la suite exacte inférieure du théorème. L'image de $W_{0}$ dans ${ }_{\nu} H_{F} / H_{c}$ est alors l'image réciproque de ${ }_{N} H_{F} / H_{f}$ par l'application naturelle, c'est-à-dire ${ }_{N} H_{F} / H_{c}$, ce qui montre aussi la suite exacte supérieure. Il reste donc à déterminer le conoyau $X$ de la flèche $V_{0} \longrightarrow{ }_{\nu} H_{F} / H_{f}$. Or en prolongeant vers la droite la première ligne de $(2 b)$, on retrouve le début de la suite exacte $(D S R)$ (voir la remarque en fin du §2.3), c'est-à-dire qu'on obtient un diagramme commutatif :

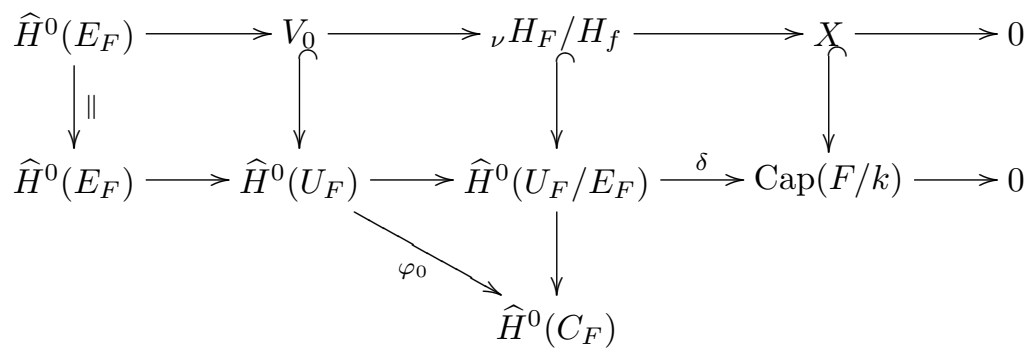

dans lequel l'injectivité de $X \longrightarrow \operatorname{Cap}(F / k)$ provient par le lemme du serpent de l'inclusion $\operatorname{Im} \varphi_{0} \subset$ $\operatorname{Im}\left(\widehat{H}^{0}\left(U_{F} / E_{F}\right) \longrightarrow \widehat{H}^{0}\left(C_{F}\right)\right)$. Soit $\gamma$ l'application composée ${ }_{\nu} H_{F} / H_{f} \longrightarrow \widehat{H}^{0}\left(U_{F} / E_{F}\right) \longrightarrow$ $\operatorname{Cap}(F / k)$. On a vu au début du $\S 2.1$ que ${ }_{\nu} H_{F} /{ }_{N} H_{F}$ s'injecte dans $\operatorname{Cap}(F / k)$, et un simple calcul de cocycles permet alors d'identifier $\gamma$ à la surjection naturelle ${ }_{\nu} H_{F} / H_{f} \rightarrow{ }_{\nu} H_{F} /{ }_{N} H_{F}$. Comme

$$
\operatorname{Ker}\left({ }_{\nu} H_{F} / H_{f} \longrightarrow X\right)=\operatorname{Ker} \gamma
$$

il s'ensuit que $X \simeq{ }_{\nu} H_{F} /{ }_{N} H_{F}$. On a bien une suite exacte

$$
\widehat{H}^{0}\left(E_{F}\right) \longrightarrow V_{0} \longrightarrow_{N} H_{F} / H_{f} \longrightarrow 0
$$

2.3. Comparaison des genres :- La puissance du théorème 2.2 se mesure au nombre de résultats plus ou moins classiques qu'il permet de retrouver. Commençons par des formules d'indice. Dans toute la suite, on posera

$$
\begin{gathered}
T_{0}=\operatorname{Im}\left(\widehat{H}^{0}\left(E_{F}\right) \longrightarrow W_{0}\right)=E_{k} N F^{\times} / N F^{\times} \simeq E_{k} / E_{k} \cap N F^{\times} \\
S_{0}=\operatorname{Im}\left(\widehat{H}^{0}\left(E_{F}\right) \longrightarrow V_{0}\right)=E_{k} N U_{F} / N U_{F} \simeq E_{k} / E_{k} \cap N U_{F}=E_{k} / E_{k} \cap N J_{F}
\end{gathered}
$$

Corollaire 2.3. - (comparer à [4], thm.2)

$$
H_{f} / H_{c} \simeq k^{\times} \cap N J_{F} /\left(E_{k} \cap N J_{F}\right) N F^{\times}
$$


Preuve : Le théorème 2.2 peut se réécrire :

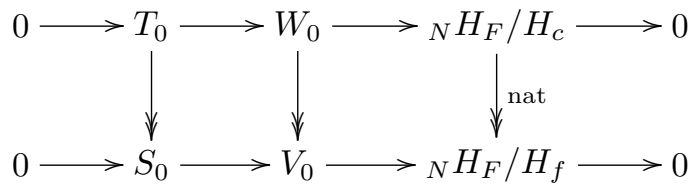

Par définition de $W_{0}$, on a $\operatorname{Ker}\left(W_{0} \longrightarrow V_{0}\right)=\operatorname{Ker}\left(\widehat{H}^{0}\left(F^{\times}\right) \longrightarrow \widehat{H}^{0}\left(J_{F}\right)\right)$, et le lemme du serpent donne immédiatement le corollaire .

On retrouve aussi les formules d'indice de Furuta :

\section{Corollaire 2.4. -}

- formule des genres:

$$
\left(H_{F}: H_{f}\right)=\frac{\left|C l_{k}\right| \prod_{v \in P l_{k}} e_{v}^{a b}}{\left[F^{a b}: k\right]\left(E_{k}: E_{k} \cap N J_{F}\right)},
$$

où $e_{v}^{a b}$ désigne l'indice de ramification en $v$ de la sous-extension abélienne maximale $F^{a b} / k$

- formule des classes centrales :

$$
\left(H_{F}: H_{c}\right)=\frac{\left|C l_{k}\right|\left(k^{\times} \cap N J_{F}: N F^{\times}\right) \prod_{v \in P l_{k}} e_{v}^{a b}}{\left[F^{a b}: k\right]\left(E_{k}: E_{k} \cap N J_{F}\right)}
$$

(dans le cas cyclique, on retrouve la formule des classes ambiges)

Preuve : Les deux formules se déduisant l'une de l'autre par l'indice $\left(H_{f}: H_{c}\right)$ (corollaire 2.3), on montrera seulement la formule des genres. D'après le théorème 2.2 :

$$
\left|V_{0}\right|=\frac{\left|S_{0}\right|{ }_{N} H_{F} \mid}{\left|H_{f}\right|}=\frac{\left|S_{0}\right|\left|H_{F}\right|}{\left|N H_{F}\right|\left|H_{f}\right|} .
$$

Or

$$
\left|V_{0}\right|=\left|\operatorname{Ker} \varphi_{0}\right|=\frac{\left|\widehat{H}^{0}\left(U_{F}\right)\right| \mid \text { Coker } \varphi_{0} \mid}{\left|G^{a b}\right|}=\frac{\prod_{v} e_{v}^{a b}\left|C l_{k}\right|}{\left[F^{a b}: k\right]\left|N C l_{F}\right|},
$$

d'où la formule pour $\left(H_{F}: H_{f}\right)$.

On peut aussi redémontrer facilement le théorème principal de Gold ([3]) :

Corollaire 2.5. - Les genres principaux coïncident : $H_{f}=H_{g}$.

Preuve : Si l'on identifie $\widehat{H}^{0}\left(F_{w}^{\times}\right)$et $G_{w}^{a b}$ par le corps de classes local, notre $S_{0}$ s'identifie au $S=\chi\left(E_{k}\right)$ qui intervient dans la définition du genre $H_{g}$, et la flèche $\psi$ dans cette définition s'identifie à la composée

$$
{ }_{N} H_{F} \stackrel{\operatorname{can}}{\longrightarrow}{ }_{N} H_{F} / H_{c} \stackrel{\sim}{\longrightarrow} W_{0} / T_{0} \longrightarrow V_{0} / S_{0} .
$$

Donc $H_{g}=\operatorname{Ker} \psi$ s'identifie à l'image réciproque de $\operatorname{Ker}\left(W_{0} / T_{0} \longrightarrow V_{0} / S_{0}\right) \simeq H_{f} / H_{c}$, i.e. $H_{g}=H_{f}$.

Remarque : Les formules précédentes font intervenir des groupes normiques usuellement appelés "noeuds" en théorie algébrique des nombres (pour un exposé synthétique, voir [7]), et qui sont fortement reliés au principe de Hasse, pour le groupe multiplicatif comme pour le groupe des unités de $k$ (voir e.g. [4]). 
2.4. La suite exacte de G. Gras :- On va retrouver fonctoriellement la suite exacte (GG) de l'introduction, dont son auteur estime qu'elle "résume entièrement la théorie des genres" pour l'extension $F / k([5]$, p.390)

Corollaire 2.6. - On a une suite exacte

$$
1 \longrightarrow E_{k} / E_{k} \cap N J_{F} \longrightarrow \widehat{H}^{0}\left(U_{F}\right) \longrightarrow \operatorname{Gal}\left(F_{h}^{a b} / k_{h}\right) \longrightarrow 1
$$

Preuve : D'après le schéma d'extensions (5), on a une suite exacte de groupes de Galois :

$$
1 \longrightarrow \operatorname{Gal}\left(F_{h}^{a b} / F^{a b} k_{h}\right) \simeq{ }_{N} H_{F} / H_{f} \longrightarrow \operatorname{Gal}\left(F_{h}^{a b} / k_{h}\right) \longrightarrow \operatorname{Gal}\left(F^{a b} k_{h} / k_{h}\right) \simeq \operatorname{Im} \varphi_{0} \longrightarrow 1
$$

qu'on veut comparer à la suite exacte déduite du théorème 2.2 et de la définition de $\varphi_{0}$ :

$$
0 \longrightarrow V_{0} / S_{0} \simeq{ }_{N} H_{F} / H_{f} \longrightarrow \widehat{H}^{0}\left(U_{F}\right) / S_{0} \stackrel{\varphi_{0}}{\longrightarrow} \operatorname{Im} \varphi_{0} \longrightarrow 0
$$

Ces deux suites montrent déjà que $\operatorname{Gal}\left(F_{h}^{a b} / k_{h}\right)$ et $\widehat{H}^{0}\left(U_{F}\right) / S_{0}$ ont le même ordre. Définissons $\phi_{0}: \widehat{H}^{0}\left(\mathcal{G}, U_{F_{h}}\right) \longrightarrow \mathcal{G}^{a b}$ de façon analogue à $\varphi_{0}: \widehat{H}^{0}\left(G, U_{F}\right) \longrightarrow G^{a b}$. Comme $k_{h} / k$ est la sousextension non ramifiée maximale de $F_{h}^{a b} / k$, on a $: \operatorname{Im} \phi_{0}=\operatorname{Gal}\left(F_{h}^{a b} / k_{h}\right)$. Par ailleurs, puisque $F_{h} / F$ est non ramifiée, la surjection canonique $\widehat{H}^{0}\left(\mathcal{G}, U_{F_{h}}\right) \longrightarrow \widehat{H}^{0}\left(G, U_{F}\right)$ est un isomorphisme (toute unité locale de $F$ est norme d'une unité locale de $F_{h}$ ). On en déduit une surjection canonique $\widehat{H}^{0}\left(G, U_{F}\right) \rightarrow \operatorname{Im} \phi_{0}$, qui passe au quotient par $S_{0}$ à cause de la formule du produit pour les unités globales. On obtient ainsi une surjection $\widehat{H}^{0}\left(G, U_{F}\right) / S_{0} \rightarrow \operatorname{Im} \phi_{0}$, qui est un isomorphisme à cause de l'égalité des ordres.

Remarque : Essentiellement, la seule différence avec la démonstration de [5] est qu'ici, on n'utilise pas les formules d'indice, mais directement le théorème 2.2. Inversement la connaissance de $(G G)$ permet de sauter une étape dans la preuve du théorème 2.2 .

2.5. La suite exacte de Karoubi-Lambre :- La suite exacte (KL) de l'introduction est une suite de Mayer-Vietoris associée à $K_{0}(N)$, où $N$ est le foncteur "restriction des scalaires" : $\operatorname{Proj}\left(\mathcal{O}_{F}\right) \longrightarrow \operatorname{Proj}\left(\mathcal{O}_{k}\right)$. Mais ce groupe $K_{0}(N)$ possède une description purement algébrique :

$$
K_{0}(N) \simeq \frac{\left\{(t, \mathfrak{A}) \in k^{\times} \times I_{F} \mid t \mathcal{O}_{k}=N(\mathfrak{A})\right\}}{\left\{\left(N(z), z \mathcal{O}_{F}\right), z \in F^{\times}\right\}}
$$

([8], théo.2-5), à partir de laquelle on peut retrouver directement (i.e. sans K-théorie) la suite (KL). Essentiellement, il suffit d'utiliser le lemme du serpent pour l'application norme à partir de la suite exacte évidente :

$$
1 \longrightarrow E_{F} \longrightarrow F^{\times} \times U_{F} \stackrel{\mu}{\longrightarrow} J_{F} \longrightarrow C l_{F} \longrightarrow 1
$$

avec $\mu(z, t)=z t^{-1}$. Heuristiquement :

- si l'on fait l'hypothèse simplificatrice que $C l_{F}$ est trivial, le lemme du serpent appliqué aux suites (6) écrites aux niveaux de $F$ et $k$, donne immédiatement la suite (KL) simplifiée :

$$
\begin{gathered}
1 \rightarrow{ }_{N} E_{F} \rightarrow{ }_{N} F^{\times} \times{ }_{N} U_{F} \rightarrow{ }_{N} J_{F} \rightarrow \widehat{H}^{0}\left(E_{F}\right)=K_{0}(N) \\
\rightarrow \widehat{H}^{0}\left(F^{\times}\right) \times \widehat{H}^{0}\left(U_{F}\right) \stackrel{\mu_{0}}{\rightarrow} \widehat{H}^{0}\left(J_{F}\right) \rightarrow C l_{k},
\end{gathered}
$$

avec $\mu_{0}(x, y)=h_{0}(x) / j_{0}(y)$, où $h_{0}$ et $j_{0}$ sont les morphismes définis au début du $\S 2$.

- dans le cas général, il faudrait couper la suite (6) à quatre termes en deux suites exactes courtes, d'où des complications dans l'application du lemme du serpent. Ce sont ces complications que l'introduction du groupe $K_{0}(N)$ permet de lever élégamment. En laissant les détails des calculs au lecteur, contentons-nous de réécrire la suite (KL) :

$$
\begin{aligned}
& 1 \rightarrow{ }_{N} E_{F} \rightarrow{ }_{N} F^{\times} \times{ }_{N} U_{F} \stackrel{\mu_{F}}{\rightarrow}{ }_{N} J_{F} \stackrel{\delta}{\rightarrow} K_{0}(N) \\
& \stackrel{\tau}{\rightarrow} \widehat{H}^{0}\left(F^{\times}\right) \times \widehat{H}^{0}\left(U_{F}\right) \stackrel{\mu_{0}}{\rightarrow} \widehat{H}^{0}\left(J_{F}\right) \rightarrow C l_{k} / N C l_{F} .
\end{aligned}
$$

Les liens avec le groupe de classes sont concentrés : 
- d'une part dans la partie droite de (KL), à savoir

$$
\widehat{H}^{0}\left(F^{\times}\right) \times \widehat{H}^{0}\left(U_{F}\right) \stackrel{\mu_{0}}{\rightarrow} \widehat{H}^{0}\left(J_{F}\right) \rightarrow C l_{k} / N C l_{F} .
$$

- d'autre part en $K_{0}(N)$, qui apparaît comme une extension naturelle

$$
0 \longrightarrow \widehat{H}^{0}\left(E_{F}\right) \stackrel{\sigma}{\longrightarrow} K_{0}(N) \stackrel{\rho}{\longrightarrow}{ }_{N} C l_{F} \longrightarrow 0,
$$

de sorte qu'on a un "carrefour"

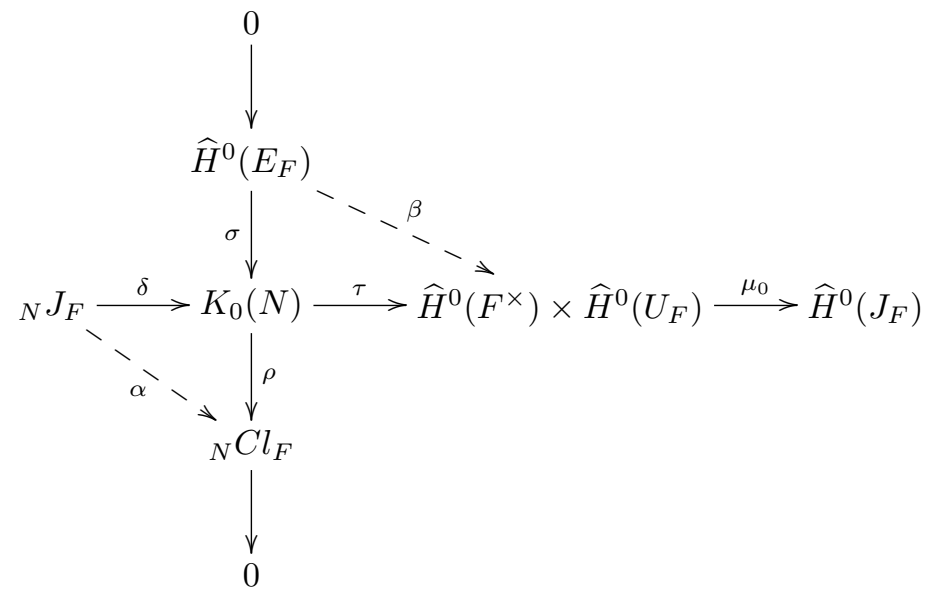

Les flèches $\alpha$ et $\beta$ sont les flèches naturelles, $\alpha=\rho \delta, \beta=\tau \sigma$, et l'on vérifie facilement, par chasse dans le diagramme, que Coker $\alpha \simeq \operatorname{Ker} \mu_{0} / \operatorname{Im} \beta$ ([8], 2-13). D'après (2b), $j_{0}$ induit un isomorphisme $\operatorname{Ker} \varphi_{0} \stackrel{\sim}{\longrightarrow} \operatorname{Ker} \mu_{0}$, et dans le cas cyclique, Karoubi et Lambre montrent que Coker $\rho \delta \simeq{ }_{N} C l_{F} / I_{G} C l_{F}$. Le théorème 2.2 et les considérations faites autour permettent de généraliser et renforcer ces résultats :

Corollaire 2.7. - Dans le cas général:

$$
\text { Coker } \mu_{0} \simeq C l_{k} / N C l_{F} \quad \text { et } \quad \text { Coker } \alpha \simeq{ }_{N} H_{F} / H_{f} .
$$

Preuve: Dans le diagramme (2b), notons $\lambda_{0}$ le composé des deux homomorphismes surjectifs $\widehat{H}^{0}\left(J_{F}\right) \rightarrow \widehat{H}^{0}\left(C_{F}\right)$ et $\widehat{H}^{0}\left(C_{F}\right) \simeq G^{a b} \rightarrow$ Coker $\varphi_{0} \simeq C l_{k} / N C l_{F}$ (voir le début du §2.1). Comme le noyau du premier (resp. second) morphisme est $\operatorname{Im} h_{0}\left(\operatorname{resp} . \operatorname{Im} \varphi_{0}\right)$, et comme $\mu_{0}(x, y)=$ $h_{0}(x) / j_{0}(y)$, il est clair que Ker $\lambda_{0}=\operatorname{Im} \mu_{0}$, et donc Coker $\mu_{0} \simeq \operatorname{Im} \lambda_{0}=$ Coker $\varphi_{0}$. Par ailleurs, Coker $\alpha \simeq \operatorname{Ker} \mu_{0} / \operatorname{Im} \beta \simeq V_{0} / \operatorname{Im} \beta$, et le théorème 2.2 permet de conclure.

Remarque : Dans le cas cyclique, on sait que $H_{f}=H_{c}$ et donc Coker $\alpha \simeq{ }_{N} C l_{F} / I_{G} C l_{F}$.

\section{Références}

[1] E. Artin and J. Tate, Class field theory, W. A. Benjamin, Inc., New York-Amsterdam, 1968.

[2] J. W. S. Cassels and A. Fröhlich (eds.), Algebraic number theory, London, Academic Press Inc. [Harcourt Brace Jovanovich Publishers], 1986, Reprint of the 1967 original.

[3] R. Gold, Genera in normal extensions, Pacific J. Math. 63 (1976), no. 2, 397-400.

[4] , The principal genus and Hasse's norm theorem, Indiana Univ. Math. J. 26 (1977), no. 1, $183-189$.

[5] G. Gras, Class field theory, Springer Monographs in Mathematics, Springer-Verlag, Berlin, 2003, From theory to practice, Translated from the French manuscript by Henri Cohen.

[6] K. Iwasawa, On cohomology groups of units for $\mathbf{Z}_{p}$-extensions, Amer. J. Math. 105 (1983), no. 1, 189-200.

[7] W. Jehne, On knots in algebraic number theory, J. Reine Angew. Math. 311/312 (1979), 215-254, In memoriam Arnold Scholz.

[8] M. Karoubi et T. Lambre, Sur la K-théorie du foncteur norme et le groupe des co-invariants du groupe des classes, prépublication (2006). 
[9] C. Maire, T-S capitulation, Théorie des Nombres, Années 1994/95-1995/96, Publ. Math. Fac. Sci. Besançon, Univ. Franche-Comté, Besançon, 1997, 1-31.

[10] D. S. Rim, An exact sequence in Galois cohomology, Proc. Amer. Math. Soc. 16 (1965), 837-840.

[11] J.-P. Serre, Corps locaux, Publications de l'Institut de Mathématique de l'Université de Nancago, VIII, Actualités Sci. Indust., No. 1296. Hermann, Paris, 1962.

16 décembre 2006

Thong Nguyen Quang Do, Université de Franche-Comté, Laboratoire de mathématiques UMR 6623,16 route de Gray, 25030 Besançon cedex, FRANCE • E-mail : nguyen@math.univ-fcomte.fr 